\title{
ON THE DUAL INTERPRETATION OF ZERO-CURVATURE FRIEDMANN-ROBERTSON-WALKER MODELS
}

\author{
L. Herrera* \\ Área de Física Teórica \\ Facultad de Ciencias \\ Universidad de Salamanca \\ 37008, Salamanca, Spain. \\ A. Di Prisco ${ }^{\dagger}$ \\ Departamento de Física \\ Facultad de Ciencias \\ Universidad Central de Venezuela \\ Caracas, Venezuela. \\ J. Ibáñez \\ Departamento de Física Teórica \\ Universidad del País Vasco, Apdo.644, \\ 48080 Bilbao, Spain.
}

\footnotetext{
*Also at Departamento de Física, U.C.V., Venezuela; email: lherrera@gugu.usal.es ${ }^{\dagger}$ On leave; email: lherrera@gugu.usal.es

‡email: wtpibmej@lg.ehu.es
} 


\begin{abstract}
Two possible interpretations of FRW cosmologies (perfect fluid or dissipative fluid) are considered as consecutive phases of the system. Necessary conditions are found, for the transition from perfect fluid to dissipative regime to occur, bringing out the conspicuous role played by a particular state of the system (the "critical point").
\end{abstract}

\title{
1 Introduction
}

It is already a well stablished fact that a variety of line elements may satisfy the Einstein equations for different (physically meaningful) stress-energy tensors (see [1] and references therein).

Particularly interesting is the situation with Friedmann-Robertson-Walker (FRW) models, which, as it has been shown ([2], [3], and references therein), do not necessarily represent perfect fluid solutions, but can also be exact solutions for viscous and heat conducting fluids, with or without an electromagnetic field.

Thus, for example, it has been shown [2], that the zero-curvature FRW metric

$$
d s^{2}=-d t^{2}+R^{2}(t)\left(d r^{2}+r^{2} d \theta^{2}+r^{2} \sin ^{2} \theta d \phi^{2}\right)
$$

satisfies Einstein equations, not only for perfect fluid matter distributions

$$
\bar{T}_{\mu \nu}=(\bar{\rho}+\bar{p}) v_{\mu} v_{\nu}+\bar{p} g_{\mu \nu}
$$

but also, for the stress-energy tensor of a magnetohydrodynamic viscous fluid with heat conducion, viz.

$$
T_{\mu \nu}=E_{\mu \nu}+(\rho+p-\xi \Theta) u_{\mu} u_{\nu}+(p-\xi \Theta) g_{\mu \nu}-2 \eta \sigma_{\mu \nu}+q_{\mu} u_{\nu}+q_{\nu} u_{\mu}
$$

where $E_{\mu \nu}, \rho, p, \xi, \eta, \Theta, \sigma_{\mu \nu}$ and $q_{\mu}$ denote respectively, the electromagnetic stress-energy tensor, the energy density, the pressure, the bulk and shear viscosity coefficients, the expansion, the shear tensor and the heat flux vector.

A fundamental difference between both interpretations of the models resides in the fact that observers moving along $v^{\mu}$ (perfect fluid case) are comoving with the fluid, whereas those moving along $u^{\mu}$ (dissipative case) detect a radial velocity of fluid particles (tilted models) [4]. In other words, in the dissipative models the source is necessarily non-comoving or tilting relative 
to the geometrically "preferred" Ricci eigenvector [3]. According to Coley and Tupper [2] this tilting velocity might be related to the observed motion of our galaxy relative to the microwave background radiation (CMB), and seems to be consistent with measurements of the dipole anisotropy of the CMB temperature distribution [3].

A detailed analysis of many of those models shows that their observational predictions are in good agreement with available data [3].

In this work, both interpretations are not considered as alternative, mutually exclusive possibilities, but rather as consecutive phases in the evolution of the model. More specifically, we shall consider the case when, initially, the system evolves as perfect fluid without dissipation, and then at some instant starts to dissipate, tending eventually again to a perfect fluid as $t \rightarrow \infty$. The question we want to answer here is: what could be the necessary condition for that transition to occur?.

For simplicity we shall consider neither electromagnetic field $\left(E_{\mu \nu}=0\right)$ nor bulk viscosity $(\xi=0)$. Furthermore, the viscosity term will be modeled through an anisotropic fluid and only transport equation for the heat flux will be needed. The reason of this simplification becomes clear later.Also, it should be kept in mind that the approach used in this work only provides a "snapshot" of the system at the moment it enters the dissipative regime, thereby giving only a hint about its tendency, and not a complete description of its evolution. All these simplifications represent the price to pay in order to deal with mathematically tractable equations.

Two different initial situations will be considered:

1. The system initially evolves strictly as a perfect fluid. By this we mean that there is not dissipation whatsoever and preferred observers (associated with the four velocity of the fluid) are (strictly) comoving with the fluid.

2. The system initially evolves very close to the perfect fluid regime, but there is still an small (in a sense to be defined below) amount of dissipation, because of which, preferred observers are not strictly comoving with the fluid, but detect an small radial motion of the fluid, so that quadratic and higher powers, as well as time derivatives, of radial velocity are neglected during this "quasi-perfect fluid" regime.

Then it is assumed that at some instant ( say $t=\tilde{t}$ ), our system starts to 
deviate from either of the initial regimes and enters into dissipative regime. In the first case the system leaves the perfect fluid condition by starting to dissipate. In the second case the system abandons the quasi-perfect fluid regime by increasing the rate of dissipation.

We shall find conditions for transitions described above to occur. With this purpose we shall evaluate field equations and transport equations immediately after $\tilde{t}$, where immediately means on a time scale of the order

of relaxation time. We shall see that the so called "critical point" plays a fundamental role in the occurrence of the transition to the dissipative regime.

The critical point [5]-[7], corresponds to the situation when (in relativistic units)

$$
\frac{\kappa T}{\tau(\rho+p)}=1
$$

where $\kappa, T$ and $\tau$ denote heat conduction coefficient, temperature and relaxation time respectively.

It has been shown [5] that under that condition, the effective inertial mass of any fluid element, just after the system departs from equilibrium, vanishes. Also, it has been shown that as a self-gravitating system approaches the critical point, its active gravitational mass behaves in such a way that enhances the instability of the system [8].

It will be shown below that transition from perfect fluid to dissipative regime is allowed only if the system is at the critical point. However, transition from the quasi-perfect fluid phase to a dissipative regime is, in principle, always possible, and closer is the system to the critical point, faster is the transition.

\section{Field and Transport equations}

\section{$2.1 \quad$ Field equations}

First of all, note that for any locally-Minkowskian observer, comoving with the fluid, the four velocity takes the obvious form

$$
\tilde{u}^{\mu}=(1,0,0,0)
$$


then, performing a Lorentz boost to the frame with respect to which a fluid element has radial velocity $\omega$, we find (in the coordinate system of (11))

$$
u^{\mu}=\left(\frac{1}{\sqrt{1-\omega^{2}}}, \frac{\omega R^{-1}}{\sqrt{1-\omega^{2}}}, 0,0\right)
$$

which is the same expression (2.1) of [2], with their $\alpha$ 's and $\beta$ 's defined in terms of $\omega$ by

$$
\begin{aligned}
& \alpha \equiv \frac{1}{\sqrt{1-\omega^{2}}}, \\
& \beta \equiv \frac{\omega}{\sqrt{1-\omega^{2}}} .
\end{aligned}
$$

Next, the heat flux vector $q^{\mu}$, satisfying $q^{\mu} u_{\mu}=0$, is given by

$$
q_{\mu}=Q\left(\frac{\omega}{\sqrt{1-\omega^{2}}}, \frac{-R}{\sqrt{1-\omega^{2}}}, 0,0\right)
$$

which is the same expression (2.3) of [2], with $Q^{2}=q_{\mu} q^{\mu}$.

Then the energy-momentun tensor (3) of the source may be writen as

$$
T_{\mu \nu}=\left(\rho+P_{\perp}\right) u_{\mu} u_{\nu}+P_{\perp} g_{\mu \nu}+\left(P_{r}-P_{\perp}\right) s_{\mu} s_{\nu}+q_{\mu} u_{\nu}+u_{\mu} q_{\nu}
$$

where $P_{r}$ and $P_{\perp}$ denote the two principal (anisotropic) stresses, linked to $p$ and $\eta \sigma_{\mu \nu}$ by relations specified below, and the vector $s_{\mu}$ satisfies conditions

$$
s^{\mu} s_{\mu}=1 \quad ; \quad s^{\mu} u_{\mu}=0 \quad ; \quad T_{\mu \nu} s^{\mu} s^{\nu}=P_{r}
$$

Then, Einstein equations read (in relativistic units and omitting the $8 \pi$ factor)

$$
\begin{aligned}
& \frac{3 \dot{R}^{2}}{R^{2}}=\frac{1}{1-\omega^{2}}\left[\rho+P_{r} \omega^{2}-2 Q \omega\right] \\
&-\left(\frac{2 \ddot{R}}{R}+\frac{\dot{R}^{2}}{R^{2}}\right)=\frac{1}{1-\omega^{2}}\left[\rho \omega^{2}+P_{r}-2 Q \omega\right] \\
&-\left(\frac{2 \ddot{R}}{R}+\frac{\dot{R}^{2}}{R^{2}}\right)=P_{\perp} \\
& 0=\left(\rho+P_{r}\right) \omega-Q\left(1+\omega^{2}\right)
\end{aligned}
$$


where dots denote derivative with respect to $t$.

Comparing (3) and (10) the following relations follow

$$
\begin{aligned}
P_{r} & =p-\frac{2 \eta}{R^{2}} \sigma_{11}\left(1-\omega^{2}\right) \\
P_{\perp} & =p+\frac{2 \eta}{R^{2}} \sigma_{11}\left(1-\omega^{2}\right) \\
\sigma_{00}=\frac{2}{3} \frac{\omega^{2}}{\left(1-\omega^{2}\right)} X & =\frac{\omega^{2} \sigma_{11}}{R^{2}}=-\frac{2 \omega^{2} \sigma_{22}}{r^{2} R^{2}\left(1-\omega^{2}\right)}=-\frac{\omega}{R} \sigma_{01}
\end{aligned}
$$

where $X$ is given by [Q]

$$
X=\frac{\omega \dot{\omega}-\omega^{2} \omega^{\prime} R^{-1}-\omega R^{-1} r^{-1}\left(1-\omega^{2}\right)}{\left(1-\omega^{2}\right)^{3 / 2}}
$$

and prime denotes derivative with respect to $r$.

Observe that from (13) and (14), one obtains

$$
\left(\rho+P_{\perp}\right) \omega^{2}+\left(P_{r}-P_{\perp}\right)-2 Q \omega=0
$$

and using (15) and (20) it follows

$$
-\frac{\left(P_{r}-P_{\perp}\right)}{\omega}+Q\left(1-\omega^{2}\right)=0
$$

Thus, if $P_{r}=P_{\perp}$ (which implies by virtue of (16)-(18), $\eta \sigma_{\mu \nu}=0$ ), then $\omega=1$, which is obviously inadmissible. Therefore, pure heat conduction (plus a fluid) is not consistent with FRW metric.

After a simple algebra, field equations (12)-(15) may be written as

$$
\begin{gathered}
\rho=\frac{3 \dot{R}^{2}}{R^{2}}+\left(P_{r}-P_{\perp}\right) \\
P_{\perp}=-\left(\frac{2 \ddot{R}}{R}+\frac{\dot{R}^{2}}{R^{2}}\right) \\
P_{r}=-\left(\frac{2 \ddot{R}}{R}+\frac{\dot{R}^{2}}{R^{2}}\right)+Q \omega \\
Q\left(1-\omega^{2}\right)=2 \omega\left(\frac{\dot{R}^{2}}{R^{2}}-\frac{\ddot{R}}{R}\right)
\end{gathered}
$$


It is worth noticing that (23)-(25) imply that dissipation takes place only if $\omega \neq 0$ and $P_{r} \neq P_{\perp}$.

Later it will be convenient to write $R(t)$ in the form

$$
R(t)=t^{n}
$$

where in general $n=n(t)$. Thus, $n=2 / 3$ reproduces the Einstein-de Sitter model, whereas

$$
n=\frac{H_{0} t}{\ln t}
$$

reproduces the exponential inflationary model with $R \sim e^{H_{0} t}, \rho+p=0$ and $p=P_{r}=P_{\perp}$.

Using (26), field equations (22)-(25) become

$$
\begin{gathered}
\rho=3(\dot{n} \ln t)^{2}+6 n \dot{n} \frac{\ln t}{t}+\frac{3 n^{2}}{t^{2}}+\left(P_{r}-P_{\perp}\right) \\
P_{\perp}=-3(\dot{n} \ln t)^{2}-6 n \dot{n} \frac{\ln t}{t}-\frac{3 n^{2}}{t^{2}}-2 \ddot{n} \ln t-\frac{4 \dot{n}}{t}+\frac{2 n}{t^{2}} \\
P_{r}=Q \omega-3(\dot{n} \ln t)^{2}-6 n \dot{n} \frac{\ln t}{t}-\frac{3 n^{2}}{t^{2}}-2 \ddot{n} \ln t-\frac{4 \dot{n}}{t}+\frac{2 n}{t^{2}} \\
Q\left(1-\omega^{2}\right)=\omega\left(\frac{2 n}{t^{2}}-\frac{4 \dot{n}}{t}-2 \ddot{n} \ln t\right)
\end{gathered}
$$

\subsection{Transport equations}

In order to avoid the important drawbacks of Eckart's theory, we shall use transport equations derived from the Israel-Stewart approach [9]-[11]. However, since these equations are going to be evaluated immediately after the system leaves the perfect fluid (or the quasi-perfect fluid) regime, then the truncated version of the theory leads to the same result as the full theory, and therefore the former will be used here. For the same reason, the shear viscosity contributions result in terms which are negligible in the approximation used here (see below). Therefore we shall deal only with the transport equation for the heat flux, which reads 11

$$
q^{\mu}+\tau h^{\mu \beta} u^{\gamma} q_{\beta ; \gamma}=-\kappa\left(h^{\mu \beta} T_{, \beta}+T a^{\mu}\right)
$$


where as usual, $h^{\mu \beta}$ denotes the projector onto the three space orthogonal to $u^{\mu}$, and $a^{\mu}$ denotes the four acceleration.

In terms of $n$, the only non vanishing independent component of (32) is the $r$-component, which reads

$$
\begin{aligned}
& -\frac{Q}{t^{n}\left(1-\omega^{2}\right)^{1 / 2}}+\tau\left\{-\frac{\dot{Q}}{t^{n}\left(1-\omega^{2}\right)}-\frac{Q^{\prime} \omega}{t^{2 n}\left(1-\omega^{2}\right)}\right\}= \\
& -\kappa\left\{\frac{\omega \dot{T}}{t^{n}\left(1-\omega^{2}\right)}+\frac{T^{\prime}}{t^{2 n}\left(1-\omega^{2}\right)}\right. \\
& \left.+T\left[\frac{\dot{\omega}}{t^{n}\left(1-\omega^{2}\right)^{2}}+\frac{\omega \omega^{\prime}}{t^{2 n}\left(1-\omega^{2}\right)^{2}}+\frac{\omega}{t^{n}\left(1-\omega^{2}\right)}\left(\dot{n} \ln t+\frac{n}{t}\right)\right]\right\}
\end{aligned}
$$

where prime denotes derivative with respect to $r$ (the $t$-component of (32) is just (33) multiplied by $\omega$ ).

\section{Entering into dissipative regime}

As was already mentioned, an essential feature of the dissipative regime, as implied by (25) or (31), is the non comoving nature of observers of the congruence $u^{\mu}(\omega \neq 0)$. This means that $\omega$ may be used as control variable to assess how far (or close) is the system from the perfect fluid regime.

So, let us now assume that for $t<\tilde{t}$, our system is in either the perfect fluid regime or in the quasi-perfect fluid regime. Both of which are characterized as follows:

1. Perfect fluid regime

$$
Q=\omega=0
$$

Preferred observers are strictly comoving with the fluid.

2. Quasi-perfect fluid regime

$$
\begin{gathered}
Q \approx \omega \approx O(\epsilon) \\
\dot{Q} \approx \dot{\omega} \approx \omega^{2} \approx Q \omega \approx Q^{2} \approx 0
\end{gathered}
$$

Preferred observers are not strictly comoving with the fluid, but (35) and (36) hold, i.e. within this regime the system always remains "close" to the perfect fluid phase. In both cases, of course, $r$ derivatives of $Q$ and $\omega$, are of the same order of magnitude as these quantities. 
Next, let us assume that at $t=\tilde{t}$, the system is allowed to abandon either of regimes (1. or 2.) and enter into a dissipative phase. We shall now find the conditions for these transitions to occur.

Let us start with the case 1 . Then, evaluating the transport equation immediately after the system leaves the perfect fluid regime $(t \approx \tilde{t}+O(\tau))$, we obtain that eq. (33) yields

$$
\dot{Q}=\frac{\kappa T}{\tau} \dot{\omega}
$$

where (34) and the fact that $T^{\prime} \approx O(\omega) \approx O(Q)$, have been used. Observe that immediately after the system leaves the perfect fluid regime

$$
P_{r}-P_{\perp}=Q \omega=O\left(\omega^{2}\right)
$$

therefore the contribution of the shear viscosity at $t=\tilde{t}$ is one order smaller than the pure heat conduction effects, and accordingly will not be considered here.

Next, it will be more convenient to use the "conservation laws" (Bianchi identities)

$$
T_{; \nu}^{\mu \nu}=0
$$

instead of field equations.

The $r$-component of (39) reads

$$
\begin{aligned}
T_{; \nu}^{r \nu}=0 & =\frac{\left(\dot{\rho}+\dot{P}_{r}\right) \omega}{t^{n}\left(1-\omega^{2}\right)}-\frac{\dot{Q}\left(1+\omega^{2}\right)}{t^{n}\left(1-\omega^{2}\right)}+\frac{\left(\rho+P_{r}\right) \dot{\omega}\left(1+\omega^{2}\right)}{t^{n}\left(1-\omega^{2}\right)^{2}}-\frac{Q 4 \omega \dot{\omega}}{t^{n}\left(1-\omega^{2}\right)^{2}} \\
& +\frac{4}{t^{n}}\left(\dot{n} \ln t+\frac{n}{t}\right)\left[\frac{\left(\rho+P_{r}\right) \omega}{1-\omega^{2}}-\frac{Q\left(1+\omega^{2}\right)}{1-\omega^{2}}\right] \\
& +\frac{1}{t^{2 n}}\left[\frac{\left(\rho+P_{r}\right) 2 \omega \omega^{\prime}}{\left(1-\omega^{2}\right)^{2}}-\frac{2 Q^{\prime} \omega}{1-\omega^{2}}-\frac{\left(1+\omega^{2}\right) 2 Q \omega^{\prime}}{\left(1-\omega^{2}\right)^{2}}\right] \\
& +\frac{2}{r t^{2 n}}\left[\frac{\left(\rho+P_{\perp}\right) \omega^{2}}{1-\omega^{2}}-\frac{Q \omega}{1-\omega^{2}}\right]
\end{aligned}
$$

which evaluated immediately after $\tilde{t}$, yields

$$
\dot{Q}=\left(\rho+P_{r}\right) \dot{\omega}
$$


Combining (37) and (41), we finally obtain

$$
\dot{\omega}\left(\rho+P_{r}\right)\left(1-\frac{\kappa T}{\tau\left(\rho+P_{r}\right)}\right)=0 .
$$

Observe that in (41) and (42) we may replace $P_{r}$ by $p$, since they differ by terms of order $O\left(\omega^{2}\right)$.

Now, since initially $(t<\tilde{t})$ the system was evolving as a perfect fluid $(\omega=Q=0)$, then a necessary condition for leaving that regime at $t=\tilde{t}$ is

$$
\frac{\kappa T}{\tau(\rho+p)}=1
$$

for otherwise $\dot{\omega}=0$, and, as can be easily checked by taking consecutive time derivatives of (33) and (40), all higher time derivatives of $\omega$ (and $Q$ ) will also vanish. As mentioned before, eq.(43) defines the critical point.

Let us now consider the case 2. In this case the system (initially) is evolving as a quasi perfect fluid, leaving that regime at $t=\tilde{t}$. Evaluating (33) at $t<\tilde{t}$, we obtain (observe that with the choice of (9), $Q>0$ implies that $q^{r}$ points inward)

$$
Q=\kappa\left[\frac{T^{\prime}}{t^{n}}+T \omega\left(\dot{n} \ln t+\frac{n}{t}\right)+\omega \dot{T}\right]
$$

then evaluating the same equation (33) immediately after $\tilde{t}$, and using (44) we get

$$
\dot{Q}=\frac{\kappa T}{\tau} \dot{\omega}
$$

as in the previous case (eq.(37)).

Next, evaluating (40) just after $\tilde{t}$ one obtains

$$
\frac{\omega\left(\dot{\rho}+\dot{P}_{r}\right)}{t^{n}}-\frac{\dot{Q}}{t^{n}}+\frac{\dot{\omega}\left(\rho+P_{r}\right)}{t^{n}}+\frac{4}{t^{n}}\left(\dot{n} \ln t+\frac{n}{t}\right)\left[\left(\rho+P_{r}\right) \omega-Q\right]=0
$$

or, using (28)-(31) and (45)

$$
\dot{\omega}\left(\rho+P_{r}\right)\left[1-\frac{\kappa T}{\tau\left(\rho+P_{r}\right)}\right]=-\omega\left(\dot{\rho}+\dot{P}_{r}\right)
$$


In the case $n=$ constant, (47) becomes (using (28) and (30) and replacing $P_{r}$ by $p$ )

$$
\dot{\omega}=\frac{2 \omega}{\left(1-\frac{\kappa T}{\tau(\rho+p)}\right) t}
$$

Therefore $\dot{\omega} \neq 0$, and is larger, the closer is the system to the critical point.

\section{Conclusions}

We have assumed in this paper that the zero-curvature FRW metric represents consecutively, first a perfect (or quasi-perfect) fluid solution, and then a dissipative solution of Einstein equations.

Necessary conditions for the transition from one state to another have been found.

If the system is initially evolving as a pure perfect fluid, then transition to the dissipative regime demands that the fluid satisfies the critical point condition. One important question related to this case is what could be the physical reasons for the system to abandon the equilibrium state?. One possible scenario might be the appearance of a dissipative process like particle creation, which as is known, is formally equivalent to the introduction of viscous terms [12]. Another possibility might be the decreasing of opacity of the fluid from very high values, preventing the propagation of null mass particles as photons and neutrinos (trapping), to smaller values allowing for radiative heat conduction and viscosity.A similar situation occurs during the gravitational collapse of stars (see [13 and references therein) and the Kelvin-Helmholtz phase of neutron stars formation (see [14 and references therein).

Of course the full implementation of any of these, or any other, scenarios would require a much more elaborated setup than the one presented here. The use of FRW with a single dissipative fluid is obviously very limiting, however as we have seen, it leads to mathematically tractable equations.

In the same order of ideas, observe that if the system is, in the initial phase, inflating exponentially with $\rho+P_{r}=0$, then as it follows from (41) and (45), it will not leave that regime as long as $\rho+P_{r}=0$. This is an obvious consequence from (28)-(31) which implies $Q=0$ if $\rho+P_{r}=0$, parenthetically it is worth noticing that the exit from inflation is usually 
explained in terms of dissipative process like particle production. From the above, it follows that though the obtained results are independent on the equation of state, some specific cases are not allowed since they exclude the possibility of dissipation (e.g. $\rho+P_{r}=0$ ).

If the system is allowed to evolve initially as a quasi-perfect fluid, then transition to dissipative regime may always occur, without requiring the critical point to be attained, however such transition will be "faster", the closer is the system to the critical point.

Another important question to ask is if real physical systems may reach the critical point. Indeed, causality and stability requirements obtained from a linear perturbative scheme are violated, close to, but below the critical point (in the absence of viscosity) [6]. On the other hand however, examples of fluids attaining the critical point and exhibiting reasonable physical properties, have been presented elsewhere [7], [15].

The possible solution to this apparent contradiction being the non-validity of the linear scheme used to obtain causality and stability conditions, close to the critical point (see a discussion on this point in [6]). At any rate, in presence of both, heat conduction and viscosity, the corresponding critical point is beyond causality and stability conditions [7].

\section{Acknowledgments}

The authors are indebted to an anonymous referee for detecting an important flaw in an earlier version of this manuscript. This work was partially supported by the Spanish Ministry of Education under grant PB96-0250,and PB96-1306.

\section{References}

[1] Tabensky R. and Taub A., 1973 Commun. Math. Phys., 29, 61; Tupper B.O.J., 1981 J. Math. Phys., 22, 2666; Tupper B.O.J., 1983 Gen. Rel. Grav., 15, 849; Raychaudhuri A.K. and Saha S.K., 1981 J. Math. Phys., 22, 2237; Raychaudhuri A.K. and Saha S.K., 1982 J. Math. Phys., 23, 2554; Carot J. and Ibáñez J., 1985 J. Math. Phys., 26, 2282.

[2] Coley A.A. and Tupper B.O.J., 1983, Astrophys. J., 271, 1. 
[3] Coley A.A., 1987, Astrophys. J., 318, 487.

[4] Triginer J. and Pavón D., 1995, Class. Quantum Grav., 12, 199.

[5] Herrera L., Di Prisco A., Hernández-Pastora J. L., Martín J. and Martínez J., 1997, Class. Quantum Grav., 14, 2239.

[6] Herrera L. and Martínez J., 1997, Class. Quantum Grav.,14, 2697.

[7] Herrera L. and Martínez J., 1998, Class. Quantum Grav., 15, 407.

[8] Herrera L. and Di Prisco A., 1999, Gen. Rel. Grav.,31, 301.

[9] Israel W., 1976, Ann. Phys., NY, 100, 310; Israel W. and Stewart J., 1979, Ann. Phys., NY, 118, 341.

[10] Jou D., Casas-Vázquez J. and Lebon G., 1999, Rep. Prog. Phys., 62, 1035 .

[11] Maartens R., 1996, Preprint astro-ph 9609119

[12] Zeldovich Ya.B., 1970. Sov.Phys.JETP Lett., 12, 307; Hu B.L., 1982, Phys.Lett.A, 90, 375; Barrow J.D., 1988, Nucl.Phys.B, 310, 743.

[13] Arnett W.D., 1977, Ap.J., 218, 815.

[14] Burrow A. and Lattimer J.M., 1986, Ap.J., 307, 178.

[15] Herrera L. and Martínez J., 1998, Astrophys. Space Sci., 259, 235. 